\title{
Considerations for the Design of UWB Antennas for Mobile and Consumer Equipment
}

\author{
Dirk Manteuffel \\ IMST GmbH, Carl-Friedrich-Gauss-Strasse 2, D-47475 Kamp-Lintfort \\ email: manteuffel@imst.de
}

\begin{abstract}
This paper presents some considerations on the design, characterization and optimization of UWB (Ultra WideBand) antennas for consumer communication equipment. As a first step a method to extract the spatio-temporal UWB antenna characterization from a FDTD simulation is given. Thereafter, the shape of a planar monopole is optimized to provide broadband matching. This is followed by the integration of latter antenna into a model of a DVD player and the impact of this integration on the antenna performance is evaluated. Finally, the transfer function of the complete system is extracted and used for indoor propagation modeling in an exemplary living home environment. The results show that the antenna integration into the DVD-chassis results into a directive radiation pattern that shows a significant frequency dependency. When this antenna is used for the propagation modeling, a single frequency raytracing simulation shows significant variation in the radiated power distribution in the room as a result of the directive pattern. When the received power is averaged over a larger bandwidth, the coverage becomes more smooth mainly due to the frequency dependency of the radiation pattern and frequency dependent propagation effects. Indeed, comparing the results of the integrated antenna to the case of the ideal isotropic radiator, no major disadvantages can be discerned anymore.
\end{abstract}

Index Terms-UWB, antennas, FDTD, transfer function, consumer equipment, propagation modeling.

\section{INTRODUCTION}

$\mathrm{T}$ he use of Ultra WideBand (UWB) systems, e.g. for wireless multimedia data communication between different home entertainment systems (DVD player, flat screen, Internet PC, ...) becomes very appealing since the FCC released the spectrum from $3.1 \mathrm{GHz}$ to $10.6 \mathrm{GHz}$ for unlicensed low-power use [1].

Especially for the design of systems entailing different antenna integration scenarios, it is essential to have fast and easy access to antenna characteristics by simple numerical simulations. This is important in the definition phase of a new product in order to predict the performance when prototypes are not yet available.

In this respect, the FDTD (Finite Difference Time Domain) method is well suited for such computations because of the fact that a large frequency spectrum can be investigated in one simulation run. Once the antenna characteristics of the device are obtained, they can be used for propagation modeling in order to predict the overall network coverage in an indoor environment.

\section{METHODS}

\section{A. UWB antenna characterization by spatio-temporal transfer functions}

From a signal processing point of view the antenna can be considered an LTI (Linear Time-Invariant) system which can be fully characterized by its transfer function [2]. This can be expressed by

$\frac{\mathbf{E}_{2}\left(\mathbf{r}_{2}, \omega\right)}{\sqrt{Z_{F 0}}}=\frac{U_{1, i n}(\omega)}{\sqrt{Z_{L}}} \mathbf{A}_{T X}\left(\hat{\mathbf{r}}_{12}, \omega\right) \frac{e^{-j k_{0} \mathrm{r}_{2}}}{\sqrt{4 \pi} r_{12}}$.

In (1) $\mathbf{E}_{2}(\mathbf{r}, \omega)$ denotes the electric field strength at a point $\mathbf{r}_{2}$ in the farfield of the antenna, positioned at location indicated by $\mathbf{r}_{1}$, which is excited by an incoming voltage $\mathrm{U}_{1, \text { in }}(\omega)$ at the antenna port (see Fig. 1). While $e^{-j k_{0} r_{2}} / \sqrt{4 \pi} r_{12}$ describes the propagation of the wave from the antenna to the observation point in the direction $\mathbf{r}_{12}, \mathbf{A}_{\mathrm{TX}}\left(\mathbf{r}_{12}, \omega\right)$ represents the transmit transfer function of the antenna. In (1) $Z_{\mathrm{F} 0}$ and $Z_{\mathrm{L}}$ are the free space and feed line impedances, respectively, and $\hat{\mathbf{r}}_{12}=\mathbf{r}_{12} / r_{12}$ is the unit vector pointing from the antenna to the observation point (farfield conditions assumed). Consequently $\mathbf{A}_{\mathrm{TX}}\left(\mathbf{r}_{12}, \omega\right)$ is independent from the distance between the antenna and the observation point but one has always to take into account that the definition of the transfer function according to (1) requires local plane wave propagation and thus is only valid if the farfield conditions apply.

On the other hand, following [2] the reception of the antenna from an incident plane wave can be expressed by

$$
\frac{U_{2, \text { out }}(\omega)}{\sqrt{Z_{L}}}=\sqrt{4 \pi} \frac{\mathbf{E}_{1, \text { inc }}}{\sqrt{Z_{F, 0}}} \mathbf{h}_{R X}(\hat{\mathbf{k}}, \omega) .
$$

In (2) $\mathrm{U}_{2, \text { out }}(\omega)$ denotes the voltage at the antenna port connected to the receiving system when the antenna is exposed to a plane wave. Note that $\mathrm{E}_{1, \text { inc }}$ is the electric field strength of the incident plane wave, i.e., the field at the 
location of the receiving antenna in absence of this antenna. According to this definition $\mathbf{h}_{\mathrm{RX}}(\mathbf{k}, \omega)$ can be considered as the receive transfer function of the antenna.

Fig. 1 and Fig. 2 illustrate the above definitions:
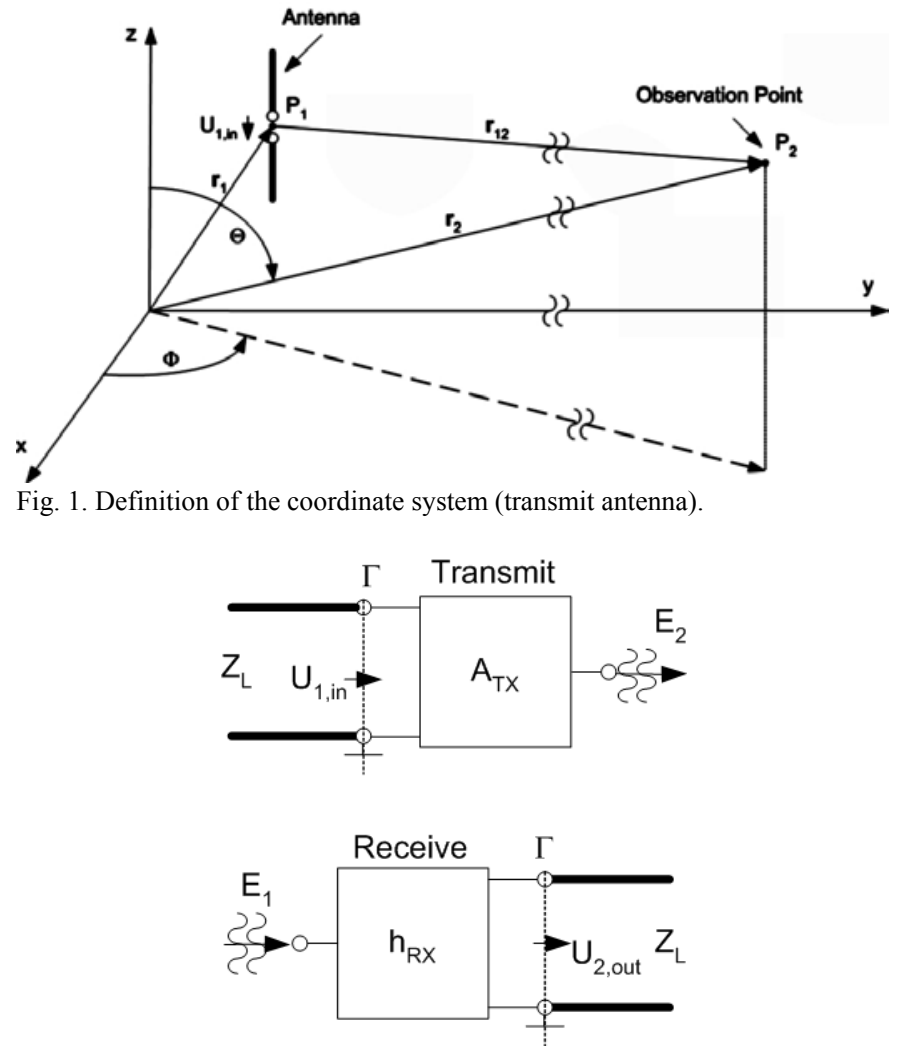

Fig. 2. Representation of the antenna as an LTI system for transmit and receive mode.

Both transmit and receive transfer functions are related to each other by Lorentz' theorem of reciprocity. An expression that takes into account the ultra wideband properties of the system has been derived in [3]:

$$
2 j \omega \mathbf{h}_{R X}(-\hat{\mathbf{k}}, \omega)=c_{0} \mathbf{A}_{T X}(\hat{\mathbf{k}}, \omega)
$$

\section{B. FDTD Simulation}

Coming back to the purpose of our investigation, this implies that it is sufficient to calculate the transmit transfer function on the basis of an FDTD simulation, for example, in order to fully characterize the antenna.

Among the numerical techniques for EM simulations the FDTD method is chosen for the following reasons:

- The FDTD method directly applies Maxwells' curl equations, and has therefore little restrictions with respect to EM-problems. Especially, it is capable of handling various types of material distribution.

- FDTD is a time-domain method; it perfectly complies to the ultra wideband behavior of the desired antennas and enables to investigate a large frequency spectrum within one simulation run.

- Due to the fact that no complex matrixes inversions have to be performed within a FDTD simulation, the memory consumption is not that demanding as in case of MoM, and therefore enables fast and efficient computation of most complex antennas using standard PCs.

The FDTD software used for this work is the 3D field solver EMPIRE $^{\mathrm{TM}}$ [4].

For the antenna characterization the FDTD simulation intends only to investigate the nearfield problem. The farfield is later determined by a nearfield-to-farfield-transformation. The technique applied here is based on Huygens' principle:

As it can be observed in

Fig. 3 the computational domain consists of the antenna and some "air-filled" space (typically less than $\lambda_{\max } / 8$ ) to the boundaries which are chosen to be a 6 layer PML (Perfectly Matched Layer) [5] in order to ensure free-space conditions. Between the boundaries and the antenna, a closed surface (a box) enclosing the antenna is defined on which the nearfield is recorded during the simulation run. Based on the recorded tangential electric and magnetic field values on the surface, equivalent electric and magnetic sources can be derived:

$$
\mathbf{S}=\hat{\mathbf{n}} \times \mathbf{H}
$$

$$
\mathbf{M}=-\hat{\mathbf{n}} \times \mathbf{E}
$$

In (4) and (5) $\mathbf{E}$ and $\mathbf{H}$ denote the electric and the magnetic field strength at a certain point on the surface, and $\mathbf{S}$ and $\mathbf{M}$ are the equivalent unit source elements. The outward directed unit vector is denoted in the above equations.
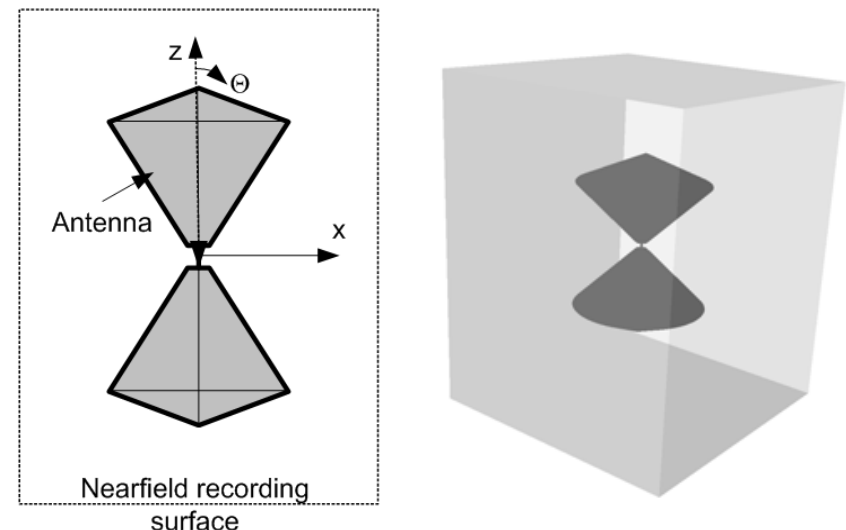

6 Layer PML Boundaries

a) General setup for the FDTD b) A biconical antenna within the simulation. $\quad$ computational domain.

Fig. 3. Illustration of the computational domain in the FDTD model showing the antenna and a box to record the nearfield for the later nearfield-to-farfieldtransformation.

\section{Validation}

In order to prove the above derived method, a biconical antenna has been analyzed. The antenna is designed to operate in the frequency range above $3.1 \mathrm{GHz}$. For the FDTD simulation the antenna is modeled with all necessary details. The distance to the PML boundaries is less than $1 / 8$ of a 
wavelength at the lowest frequency of interest thus resulting in a time and memory efficient simulation. The antenna is excited by a broadband Gaussian pulse centered at $0 \mathrm{~Hz}$, and having a half bandwidth of $20 \mathrm{GHz}$ with reference to a signal decrease of $20 \mathrm{~dB}$. The nearfield of the antenna is recorded at every $200 \mathrm{MHz}$ intervals between $1 \mathrm{GHz}$ and $20 \mathrm{GHz}$ on a Huygens' surface enclosing the antenna. The EMPIRE ${ }^{\mathrm{TM}}[4]$ field solver uses this nearfield data to derive equivalent electric and magnetic sources on the surface and extrapolate the near field into the farfield. The total simulation time, including the post-processing of the nearfield data, takes only a few minutes on a standard $2 \mathrm{GHz}$ PC. The results from this simulation are used to determine the transmit and receive transfer functions of the antenna according to the above mentioned method.

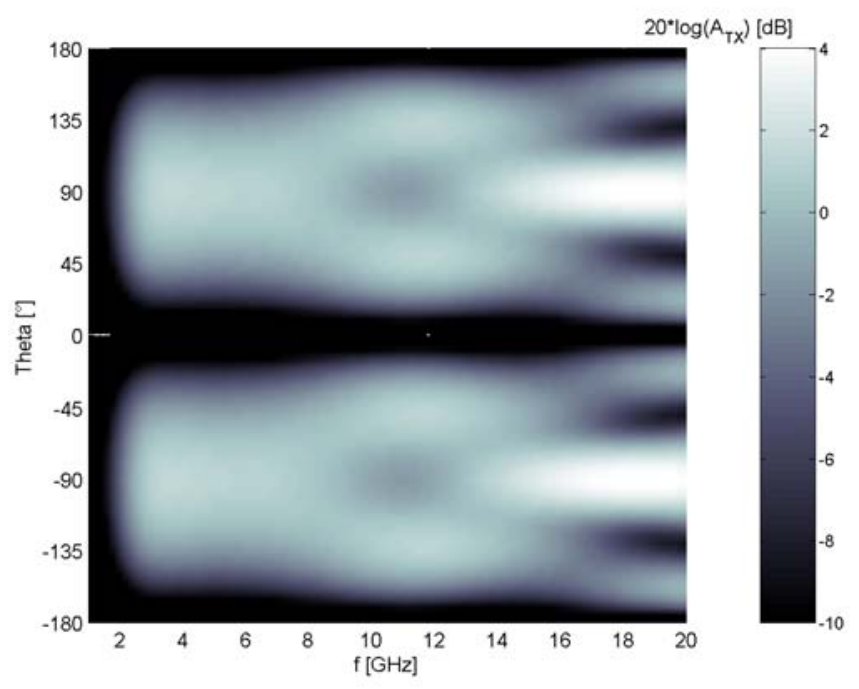

a) TX transfer function.

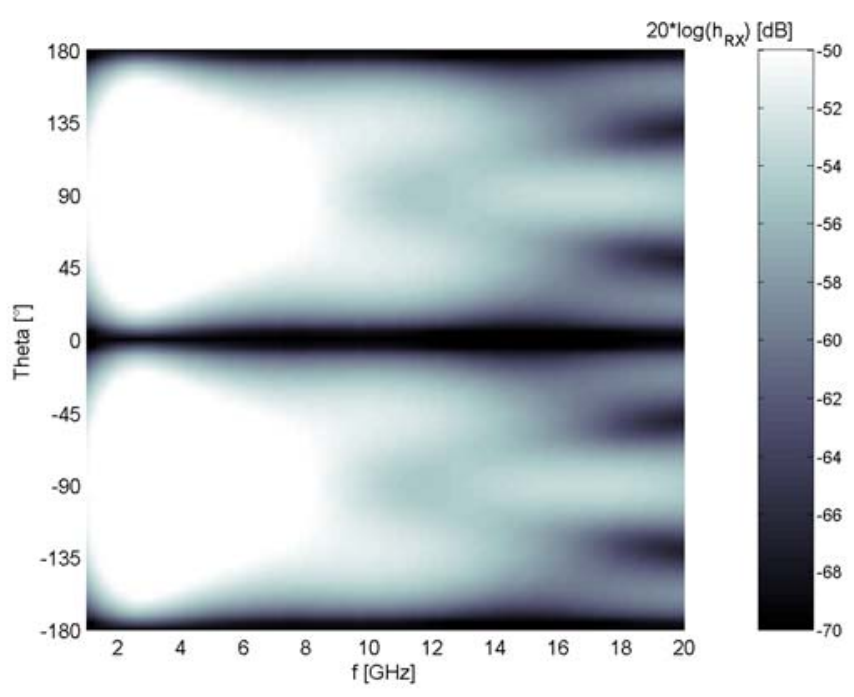

b) RX transfer function.

Fig. 4. Calculated transfer functions of the biconical antenna in the E-plane.

Fig. 4 shows the calculated transfer functions of the biconical antenna in the E-plane. The TX transfer function has been directly calculated from the FDTD simulation using equation (1). The RX transfer function is derived from the TX transfer function applying Lorentz' reciprocity principle according to equation (3). Fig. 4a illustrates the TX transfer function of the biconical antenna in the E-plane. It can be observed that the antenna is matched above $3 \mathrm{GHz}$. The characteristics are similar to those of a standard $1^{\text {st }}$ order dipole up to a frequency of $8 \mathrm{GHz}$. For higher frequencies, the characteristic changes showing sidelobes and gain deviations.

To validate the approach, a second simulation model is defined that consists of two biconical antennas separated by a distance of $\mathrm{d}=50 \mathrm{~cm}$. While the first antenna is fed by the Gaussian pulse the second antenna is passive, and receives the radiated pulse. Therefore, it is possible to calculate the transmission between both antennas in terms of $s_{21}$ using this full wave analysis. Having in mind that the FDTD method requires the discretization of not only the antennas but also the free space in-between, this results in quite a large computational model.

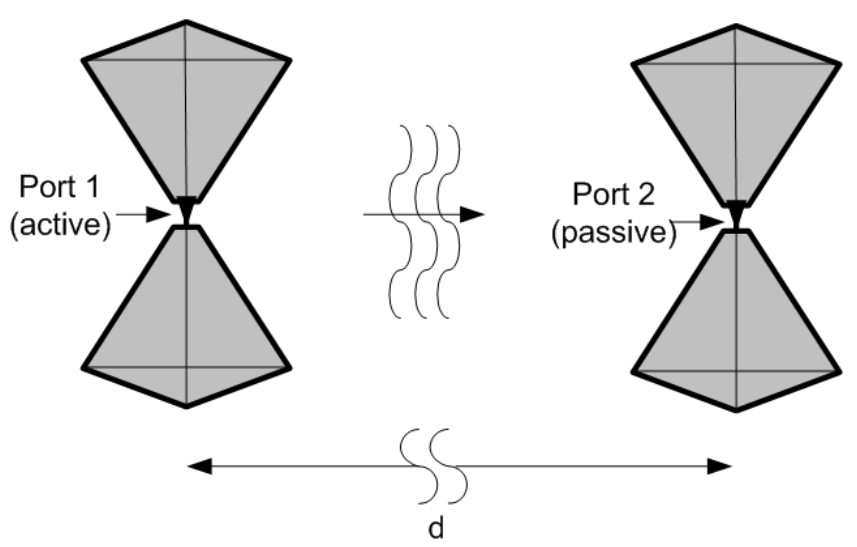

Fig. 5. Transmission between two antennas (Principle).

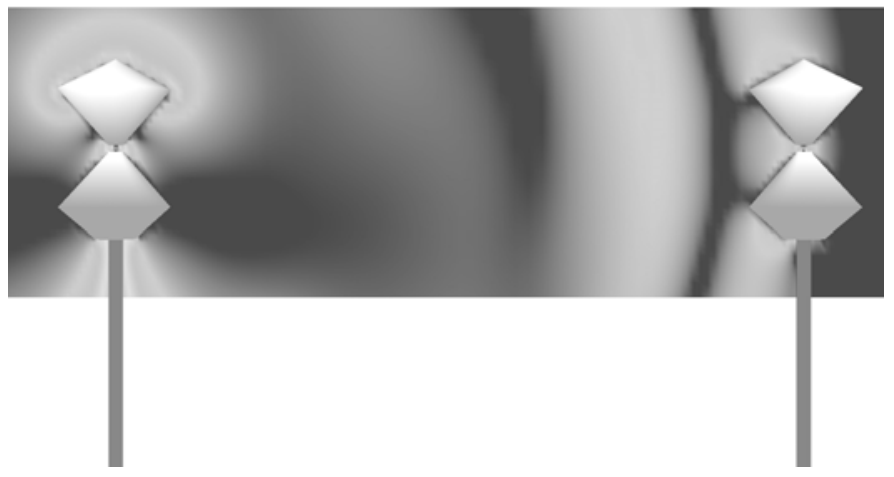

Fig. 6. Time domain computation of two-antenna system.

In addition to the direct calculation of $s_{21}$, based on the complete FDTD simulation of two antennas, we can also use the transfer functions from the one-antenna simulation and calculate the transmission between two antennas by the following expression [2]:

$$
S_{21}(\omega)=\left.\frac{b_{2}}{a_{1}}\right|_{a_{2}=0}=\mathbf{A}_{1}\left(\hat{\mathbf{k}}_{12}, \omega\right) \mathbf{h}_{2}\left(\hat{\mathbf{k}}_{12}, \omega\right) \frac{e^{-j k_{0} d}}{d}
$$


Fig. 7 compares the results from both methods which show a good agreement.

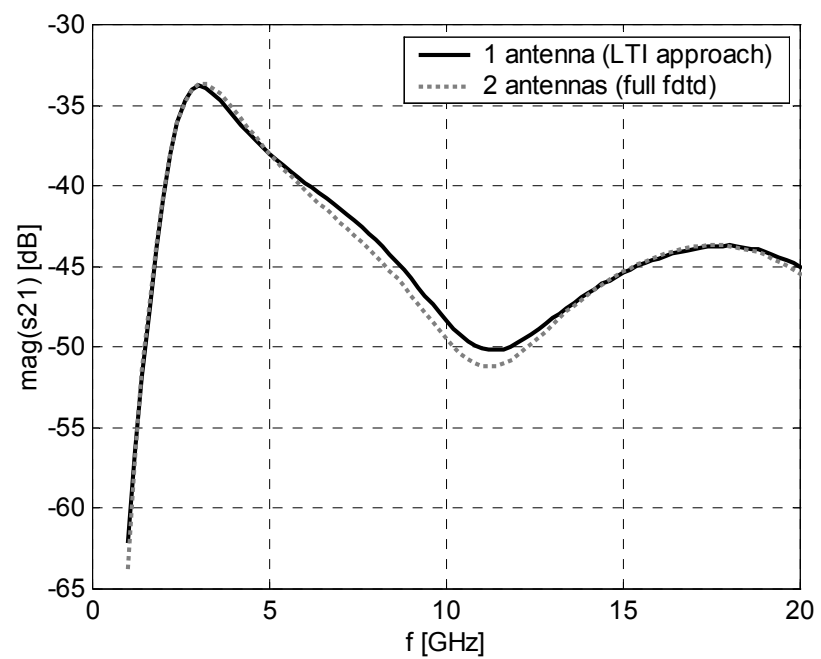

Fig. 7. Transmission between two antennas calculated by the FDTD simulation of two antennas, and the FDTD simulation of a single antenna combined with an LTI approach.

This proves that the method described above has been implemented in a correct way. In addition to the validation aspect of such a calculation, it should be noted that it is now possible to fully characterize an antenna by a single FDTD simulation of only the transmitting antenna. Furthermore, the TX- and RX-transfer functions can be used e.g. for propagation simulations, for example, using other simulation tools.

\section{ANTENNA DESIGN AND INTEGRATION}

The above developed method is used in the following section to design a broadband miniaturized antenna and integrate it into a model of a DVD player as an example for a typical device of home-entertainment systems. The problem is divided into two parts. First a miniaturized UWB antenna is designed and optimized. Afterwards this antenna is integrated into the DVD player and the influence is investigated. Although necessary for a real development, the optimization in the specific integration scenario is not performed in this paper. Finally the complete antenna characterization is extracted from the FDTD simulation for later use with other modeling tools.

\section{A. Antenna element design and optimization}

From the EM modeling point of view a suitable antenna has to be designed in the first step. Because of the intended application the following basic requirements for the antenna element can be listed:

- Large bandwidth

(e. g.: $s_{11} \leq-10 \mathrm{~dB} \forall \mathrm{f} \in\{3.1 \mathrm{GHz}, 10.6 \mathrm{GHz}\}$ ),

- small size (integration into device),

- low cost technology.

Additionally often other quality criteria like
- omni directional radiation pattern,

- frequency stability (radiation pattern),

- low ringing

are associated with and requested for UWB antennas [6]. However, of the following investigation will also evaluate if the last mentioned criteria are of great importance when we focus on a UWB communication system for the consumer market.

It is widely reported in recent antenna literature [7] that a broadband planar monopole antenna can be good candidate. The antenna shape differs from a basic wire monopole in the way that the element provides a smooth taper relative to the groundplane. By optimizing this taper broadband impedance matching can be obtained quite easily.

Fig. 8 shows some examples of basic shapes for planar broadband monopoles:

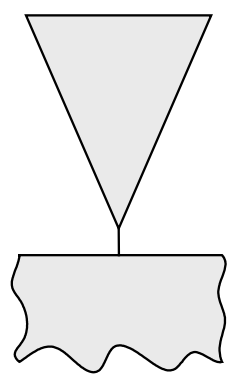

a)

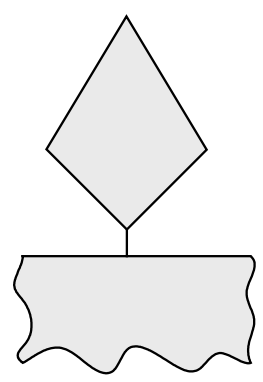

b)

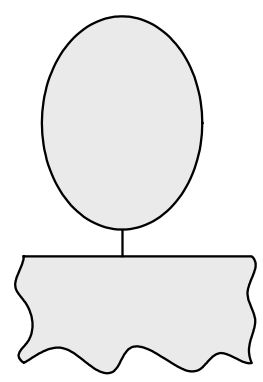

c)
Fig. 8. Different basic shapes of planar broadband monopoles on a groundplane.

In order to apply an automated optimization process for the simulation modeling it is advantageous to describe the antenna by an analytic mathematical function. This can be e.g. polynomial, elliptical, exponential or any other kind of analytical function which provides a smooth taper. In this paper the following parametric polynomial approach is used because it offers maximum flexibility in the generation of various shapes:

$$
\begin{aligned}
& x=\frac{w}{2} \sin (\pi \alpha), \\
& y=\frac{h}{2} \cos \left(\pi\left(k|\alpha|^{p_{1}}-1\right)\right)
\end{aligned}
$$

$w$ Is the maximum width of the antenna and $h$ is its maximum height, $\alpha$ ranges within $-1 \leq \alpha \leq 1$. In (8) the factor $\mathrm{k}$ is

$$
k=\frac{\cos ^{-1}\left(2 p_{2}-1\right)}{\pi}+1 .
$$

These continuous euclidic functions depend on the power $\mathrm{p}_{1}$ (see Fig. 9a) and the parameter $\mathrm{p}_{2}$ (see Fig. 9b), which enable a continuous transition into manifold antenna shapes (circle, ellipse, triangle-like, ...). 
The shape is first optimized to offer good matching when the antenna is located on a groundplane. The variables $p_{1}$ and $\mathrm{p}_{2}$ can now be assessed using the GUI of the simulator. This provides an easy way to generate a large number of different shapes for a series of simulations or the use of an optimizer.

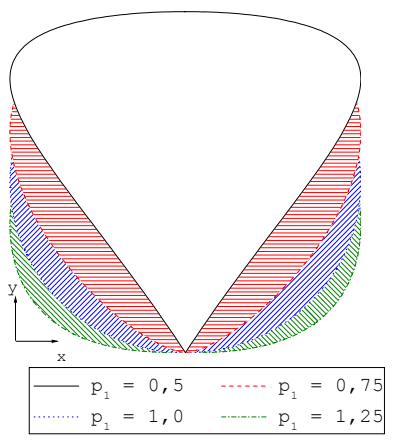

a) variation of $p_{1}$

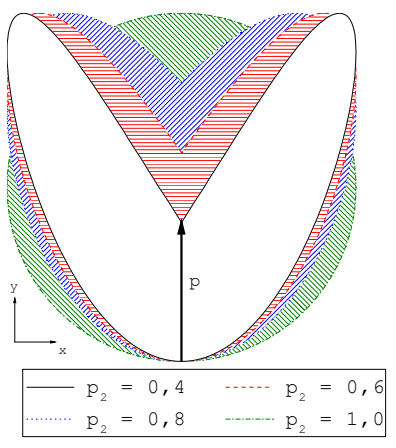

b) variation of $p_{2}$
Fig. 9. Parametric generation of various shaped.

When an optimizer is available within the simulation software, different variations are generated automatically and varied in order to approximate a certain goal function which has to be defined in advance. For our example the goal function could be:

$$
\begin{aligned}
& \operatorname{Goal}\left(p_{1}, p_{2}\right)= \\
& s_{11} \leq-10 \mathrm{~dB} \forall \mathrm{f} \in\{3.1 \mathrm{GHz}, 10.6 \mathrm{GHz}\}
\end{aligned} .
$$

Starting from a certain initial state, the parameters are now varied and after each step the approximation of the simulation result to the goal function is evaluated. The next evolution of the shape is now chosen on the basis of an estimation by a gradient approach or a genetic algorithm, depending on the optimizer. Using a prior optimised grid a single simulation takes less than a minute on a state of the art PC. Fig. 10 shows the results of two different shapes that fulfill the goal function in comparison to the initial design of the monopole.

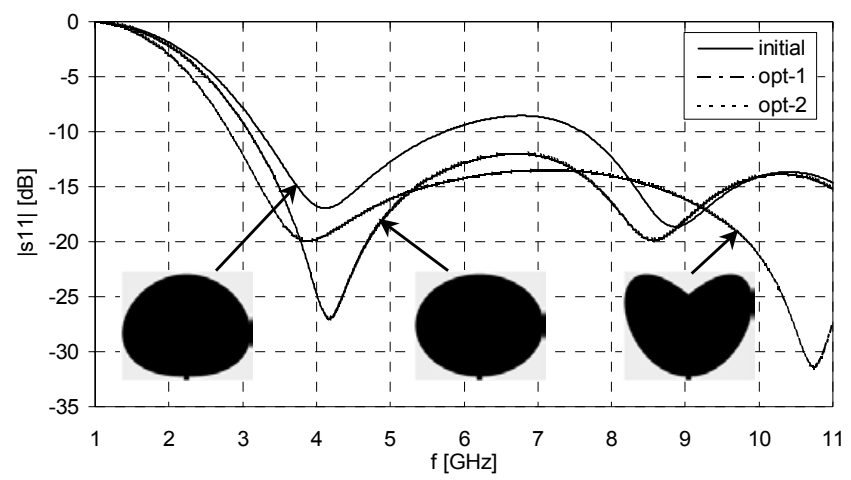

Fig. 10. Results from the parametric optimization.

\section{B. Antenna integration}

It is obvious that all antenna parameters are affected when the antenna is transferred from the groundplane to a specific situation inside a (partly) metal chassis. For a detailed analysis three different integration scenarios are observed. They are illustrated in the photograph of the DVD player shown in Fig. 11: In scenario no. 1 the antenna is mounted on the top edge of the DVD player. This is the most similar situation compared to the groundplane case that may have at least some practical relevance. A more likely case may be scenario no. 2 in which the antenna is integrated within a certain volume at the corner of the device. In scenario no. 3 the antenna is integrated within a volume inside the front face of the DVD player. Fig. 11 shows the influence of the integration volume on the matching of the antenna for scenario no. 3. It can be observed that allowing a distance of $25 \mathrm{~mm}$ to the metal walls of the surrounding structure, the antenna still fulfills the requirements in terms of matching. Note: The assumption that the DVD player is completely composed out of metal is quite pessimistic. It may be possible to find a smaller volume with less interference in a more realistic scenario. Additionally, as mentioned earlier, in an industrial development the antenna element would be optimized again for this specific integration scenario.

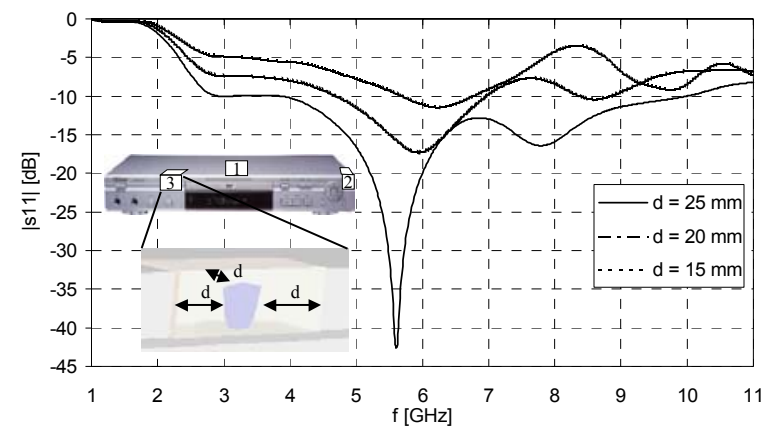

Fig. 11. Antenna integration in the front side of the DVD player: Influence of the integration volume on the antenna matching.

In the next step the influence of the different integration scenarios on the radiation pattern of the antenna is investigated. Fig. 12 shows the simulation models for the different integration scenarios together with the farfield pattern at $\mathrm{f}=7 \mathrm{GHz}$ (as an example):

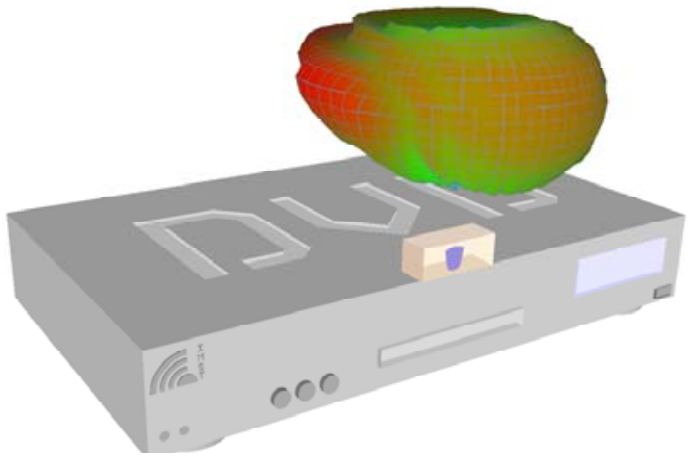

a) Antenna on top at front side. 
6

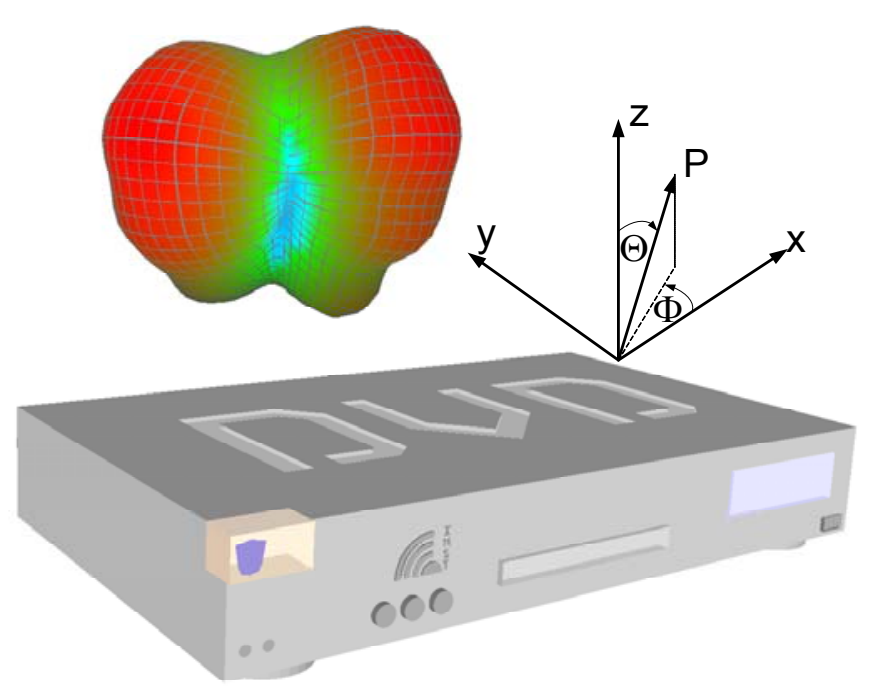

b) Antenna integrated in corner.

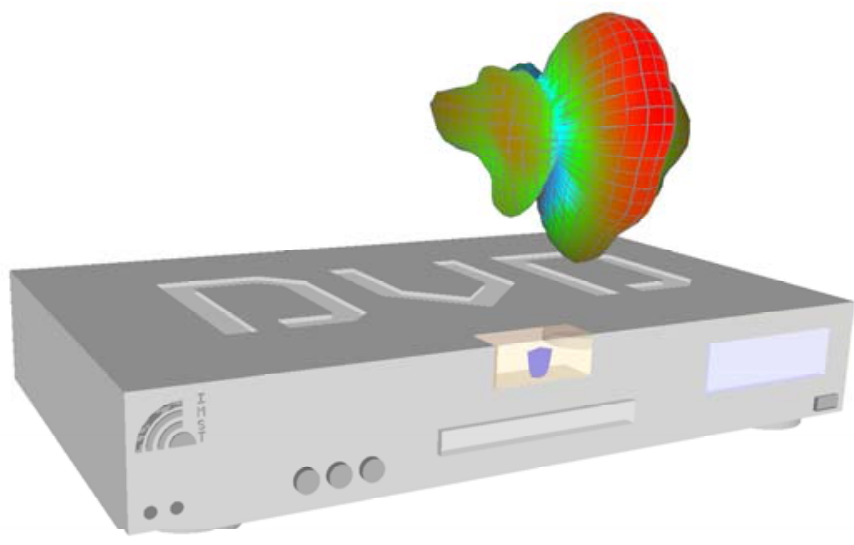

c) Antenna integrated in front side

Fig. 12. Influence of different integration scenarios on the radiation pattern (e. g. at $\mathrm{f}=7 \mathrm{GHz}$ ).

Fig. 12 shows clearly that the pattern depends very much on the specific integration scenario and is in any case no longer quasi omni-directional as it was mounted on the groundplane. Fig. 13 shows two planes of the radiation pattern of the model in Fig. 12c as a function of frequency. It can be observed that the radiation pattern shows a strong frequency dependency. The system provides a single main beam up to $6 \mathrm{GHz}$, and sidelobes occurring at higher frequencies. Furthermore, it is clearly illustrated that there are shadow areas located at the backside of the DVD player.

In general we can conclude that even if the assumption of a metal chassis with only a small integration volume for the antenna may be quite pessimistic, it is clear that we cannot reach a quasi omni-directional radiation which is stable over the frequency range when the antenna is integrated in (or even mounted on) a realistic device. Therefore, the demand for omni directionality or frequency stability that is often requested is surely not feasible for low cost UWB antennas in consumer applications. However, at this stage it is an open question if we finally need such strict antenna demands to achieve sufficient network coverage in a home environment. In order to answer these questions the broadband antenna characterization can be extracted from the FDTD simulation and used as input data for propagation modeling.

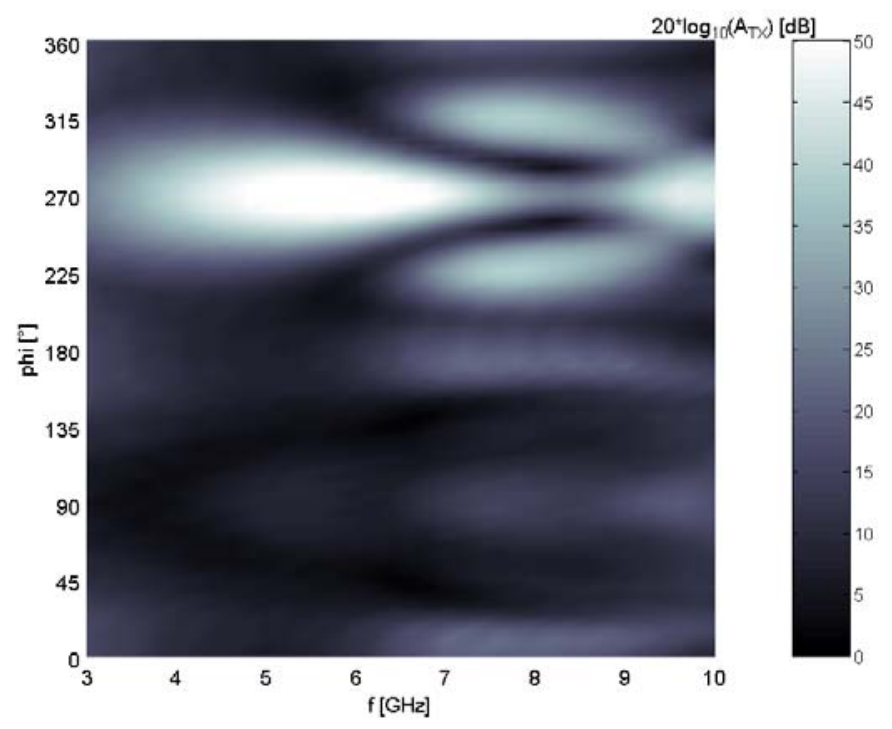

a) Horizontal plane: $\mathrm{E}\left(\phi, \theta=90^{\circ}, \mathrm{f}\right)$

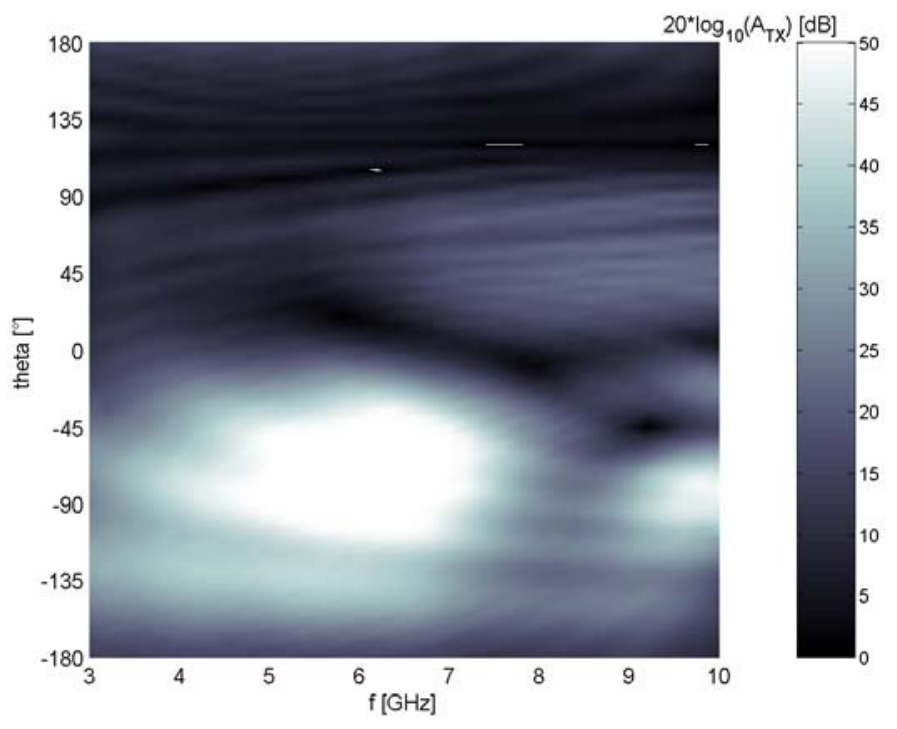

b) Vertical plane: $\mathrm{E}\left(\phi=90^{\circ}, \theta, \mathrm{f}\right)$

Fig. 13. Horizontal and vertical plane of the frequency dependent radiation patter of the scenario in Fig. 12c.

\section{Propagation Modelling}

UWB for consumer equipment is a new technology where not much experience in the design and performance of the complete system exists yet. One main fear of the system designer who decides to replace the cable by a UWB module is that certain areas in the room may not be covered. Considering only simple antenna and propagation models this may be due to nulls in the radiation pattern of the antenna, shadowing by objects in the room or fading due to reflections. The first reason is directly related to the antenna and indeed 
often quasi omni-directional radiation patterns are therefore requested by the system designer. However, based on the prior investigation we have seen that this will be impossible for a realistic integration situation. All in all it is not that simple to answer if all of these requirements are really necessary or what is the impact on the performance at all if they cannot be fulfilled completely. This can only be answered by a thorough investigation including propagation modeling using realistic radiation characteristics of the complete system. A first approach will be presented in the final step.

In order to investigate network coverage in a home environment the data is finally transferred to a ray-tracing software for propagation modeling. An artificial living room is set up. The DVD player is placed in a shelf at one side of the room. Fig. 14 shows a picture of the room including an interior.

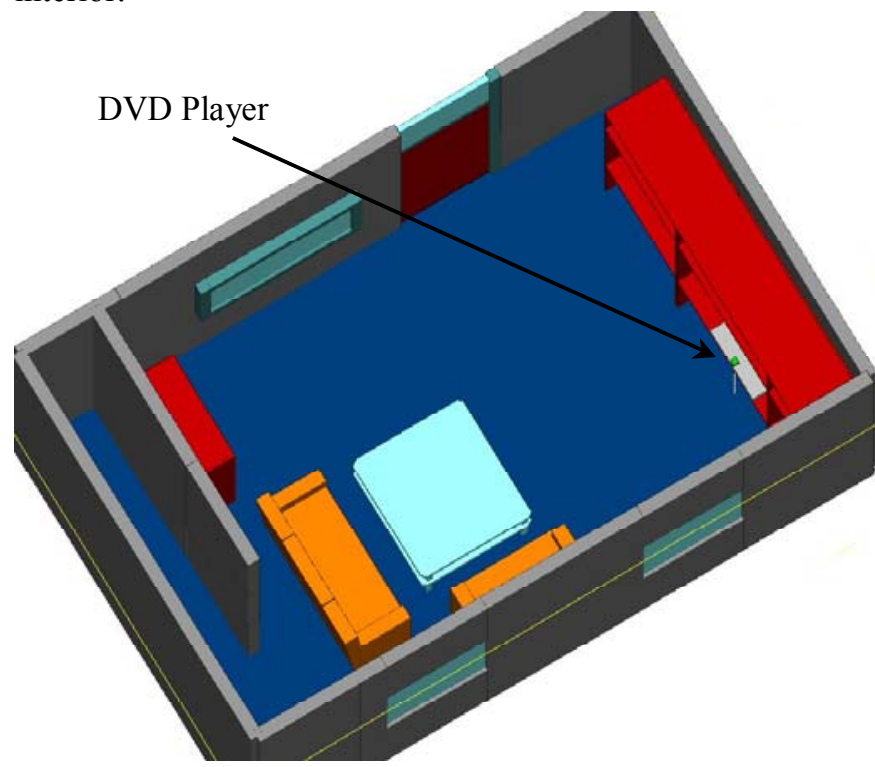

Fig. 14. Typical living room modeled using the ray-tracing tool Wireless Insite. The DVD player is positioned in a shelf at the right side of the room.

The room has been modeled using the $3 \mathrm{D}$ ray-tracing software Wireless Insite. A script for the transition from the antenna characteristics calculated using EMPIRE $^{\mathrm{TM}}$ to Wireless Insite is written within MathLab.

Fig. 15 shows a map of the power distribution in a plane of $1 \mathrm{~m}$ constant height from the floor in the living room when the DVD player with the antenna integrated according to scenario no. 3 (see Fig. 12c) is used. In order to show the effect of the directive pattern a single frequency of $f=7 \mathrm{GHz}$ is used. It can be observed from Fig. 13a in this frequency range that the antenna patterns splits into several beams. The effect of these separated beams is also clearly visible in Fig. 15. As a result some directions benefit from the higher gain, some areas suffer from being in the direction of a null in the pattern.

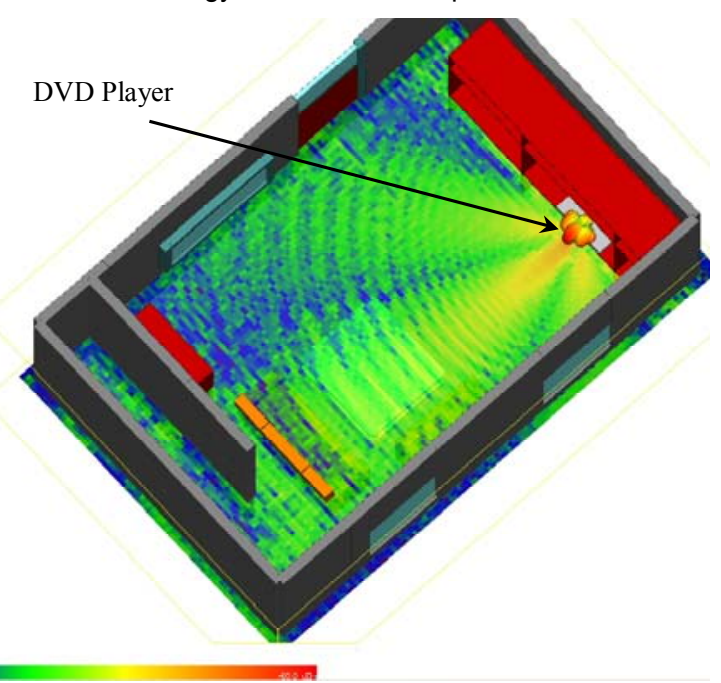

Fig. 15. Map of the power distribution in the room at $\mathrm{f}=7 \mathrm{GHz}$ using the planar monocone antenne integrated in the DVD player (scenario 3, see Fig. 12c).

Additionally the coverage is significantly affected by fading as it can be observed from the standing wave shape modulated on the pattern of the power map. Of course this would not be different for an quasi omni-directional antenna. At this stage another advantage of UWB propagation can be mentioned: It has been reported in [8] that fading becomes smaller when the bandwidth of the transmitted pulse becomes broader. In this respect a second propagation investigation has been performed. First propagation simulations at single frequencies between $3.1 \mathrm{GHz}$ and $10.6 \mathrm{GHz}$ are performed. Later the received power of all simulations is averaged over the frequency range. (Remark: The usage of the complete bandwidth between $3.1 \mathrm{GHz}$ and $10.6 \mathrm{GHz}$ is of course not intended for first systems. More realistic bandwidth will be smaller in the beginning.)

The results of this investigation are displayed in Fig. 16 first for the case of the DVD player applying again integration scenario 3 (see Fig. 16a) and later for an isotropic antenna for sake of comparison (see Fig. 16b).

It can be observed that the map of the power distribution becomes more smooth due to the averaging. In case of the DVD player this is not only because of the beneficial effect on the fading but also the frequency dependent radiation pattern (that was deemed to be unwanted in the beginning).

The comparison to the "ideal" isotropic radiator shows that only small areas at the outer regions of the room are covered less using the realistic model while the largest part of the overall room shows even better signal. Nevertheless this has to be validated more carefully, because the dynamic range of the power distribution is quite large. Therefore, further investigations will make use of more practical parameters like SNR or even by the simulation of the entire communication link between different devices. 


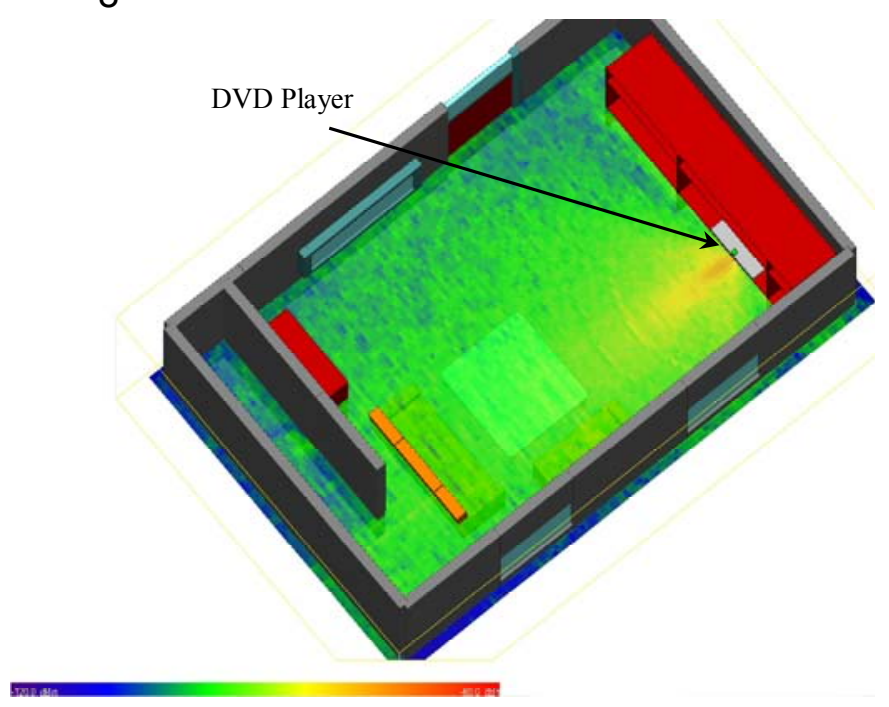

a) Monopole antenna integrated in the frontside of the DVD player (see Fig. 12c and Fig. 13).

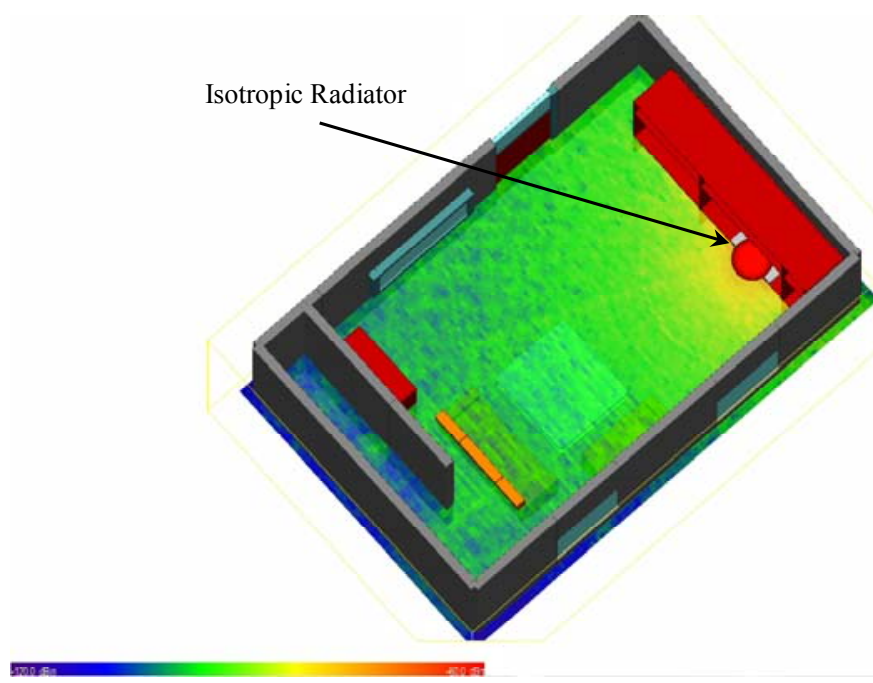

b) Isotropic antenna.

Fig. 16. Ray tracing simulation of DVD player with integrated UWB antenna in a artificial living room environment. Map of the received power averaged over a frequency span from $3.1 \mathrm{GHz}<\mathrm{f}<10.6 \mathrm{GHz}$. Comparison between the integrated antenna and an isotropic radiator.

\section{CONCLUSION}

Planar monopole antennas are good candidates for the costand size-efficient integration into the UWB module for devices of consumer equipment. The FDTD method is ideally suited for the numerical analysis and design of the entire antenna system. The antenna characterization of the system can be extracted to be used for propagation modelin,. thus enabling us to evaluate directly the coverage in a room when different antennas are used. The results show that the impact of the directive and frequency dependent radiation pattern of the integrated antenna is not that strong as one would expect. However, this has to be investigated carefully in more detail in future work.

\section{REFERENCES}

[1] Federal Communications Commission (FCC): Revision of Part 15 of the communication's rules regarding ultra wideband transmission systems. First report and order, ET Docket 98-153, FCC 02-48; Adopted: Feb. 14th, 2002; Released: Apr. 22nd, 2002.

[2] D. Manteuffel, J. Kunisch: Efficient characterization of UWB antennas using the FDTD method. AP-S - International Symposium on Antennas and Propagation, Monterey (CA) USA, June 2004.

[3] J. Kunisch, J. Pamp: UWB radio channel modeling considerations. In: Proc. Of ICEAA'03, Turin, Sep. 2003.

[4] IMST GMBH: User and Reference Manual for the 3D EM Time Domain Simulator Empire. http://www.empire.de/empire.pdf, November 2003

[5] J. P. BERENGER: A perfectly matched layer for the absorption of electromagnetic waves. In: J. Comp. Phys., vol. 114, pp. 185-200, 1994.

[6] W. Sörgel, Ch. Waldschmidt, W. Wiesbeck: Transient response of Vivaldi antenna and logarithmic periodical dipole array for ultra wideband communication. In: AP-S - International Symposium on Antennas and Propagation, Proc. on CDROM, Columbus (Ohio) USA, 2003.

[7] M. Cabedo-Fabrés, M. Ferrando-Bataller, A. Valero-Nogueira: Innovative wide-band planar monopole antennas for multi-service mobile systems. AP-S - International Symposium on Antennas and Propagation, Columbus (OH) USA, July 2003.

[8] J. Romme, B. Kull: On the relation between bandwidth and robustness of indoor UWB communication. In: UWBST Conference, Reston, Nov. 2003.

Dirk Manteuffel was born in Issum, Germany in 1970. He received the Dipl.-Ing. degree in electrical engineering from Duisburg University in 1998 and the Dr.-Ing. degree from the University of Duisburg-Essen in 2002. In 2004 he received the young scientist award from the Vodafone foundation for science for his research on the analysis and design of integrated mobile phone antennas with special emphasis on the interaction with the user.

Since 1998 he is with the IMST in Germany. As a project manager he is responsible for industrial antenna

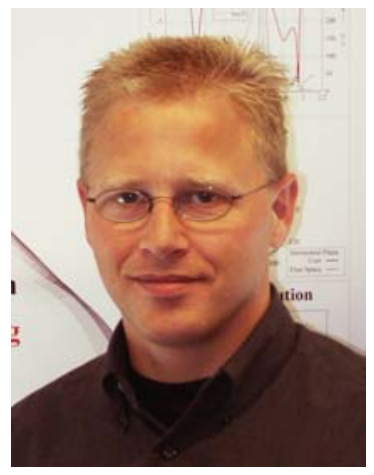
development and advanced projects in the field of antennas and EM modeling. His research interests cover terminal antennas and UWB as well as EM modeling.

Dr. Manteuffel is inventor of 6 national and international patents and author and co-author of more than 30 scientific publications. 